\title{
Lymphatic absorption of plasmalogen in rats
}

\author{
Hiroshi Hara*, Takuya Wakisaka and Yoritaka Aoyama \\ Division of Applied Bioscience, Graduate School of Agriculture, Hokkaido University, Kita-9, Nishi-9, Kitaku, \\ Sapporo 060-8589, Japan
}

(Received 9 September 2002 - Revised 20 January 2003 - Accepted 31 January 2003)

\begin{abstract}
Plasmalogen is a subclass of phospholipids that is widely distributed in man and animals. Many physiological roles have been proposed for this lipid; however, there have been no reports on the intestinal absorption of plasmalogen. In the present study, we examined lymphatic absorption of plasmalogen after the duodenal infusion of emulsified brain phospholipids (BPL) containing plasmalogen ( $22 \mathrm{~mol} \%$ of total phospholipids) and soyabean lecithin (SPL) (100 g emulsified phospholipid/l). Male Wistar rats with implanted cannulas in the mesenteric lymph duct and the duodenum were kept in a Bollman-type restraining cage, and were infused the emulsion after $1 \mathrm{~d}$ recovery with duodenal infusion of a glucose $-\mathrm{NaCl}$ solution. Lymphatic plasmalogen output was increased at $2-4 \mathrm{~h}$ after the switch to BPL emulsion, and peaked at 4-6h. However, no increases were observed after SPL infusion. Lymphatic recovery of plasmalogen for $8 \mathrm{~h}$ was $198 \mathrm{nmol}$, which was $0.22 \mathrm{~mol} \%$ of the total plasmalogen disappeared from the intestine. We did not detect any increases in long-chain fatty aldehydes, which are the degradation product of plasmalogen, either in the blood or the small intestine. We conclude that a small percentage but a significant amount of the plasmalogen was absorbed into the lymph.
\end{abstract}

Plasmalogen: Phospholipids: Lymphatic absorption: Rats

Plasmalogen has a vinyl-ether double bond in the $s n-1$ position (1-O-alk-1'-enyl) that is susceptible to oxidative molecules (Zommara et al. 1995; Zoeller et al. 1999). While the role of plasmalogen is not yet fully understood, a protective role against oxidative stress has been proposed in cerebral and cardiac tissues and plasma lipoproteins (Zoeller et al. 1988; Engelmann et al. 1994; Jurgens et al. 1995). These previous studies suggest that increases in plasmalogen levels in plasma lipoprotein protect against cholesterol oxidation and lower the incidence of CHD. Plasmalogen may be an endogenous lipidaemic antioxidant. Many reports also suggest that plasmalogen has a role in the maintenance of cell membrane dynamics as a structural component (Glaser \& Gross, 1994). Recently, Farooqui et al. (1997) reported that plasmalogen deficiency is involved in nerve degeneration in Alzheimer's disease.

Plasmalogen is widely distributed in human and animal tissues. The brain, heart, lung and erythrocytes contain relatively high levels of ethanolamine plasmalogens (Nagan \& Zoeller, 2001), and many foods also contain plasmalogen (Blank et al. 1992). Phospholipids in the diet are absorbed via the lymph; however, there are no reports on the intestinal absorption of ingested plasmalogen or on the fate of the vinyl-ether double bond in plasmalogen after ingestion. The aims of the present study were to examine the lymphatic absorption of plasmalogen extracted from the brain as glycerophospholipids, and to examine its degradation into fatty aldehydes during intestinal absorption.
Methods
Animals and diets
Male Wistar rats (Japan SLC, Hamamatsu, Japan), weigh- ing about $270 \mathrm{~g}$, were given free access to tap water and a semi-purified casein-sucrose-based stock diet (American Institute of Nutrition, 1977, 1980) for a $6 \mathrm{~d}$ acclimatization. A polyvinyl tube (SV-35, internal diameter $0.5 \mathrm{~mm}$, exter- nal diameter $0.9 \mathrm{~mm}$; Natsume Seisakusyo, Tokyo, Japan) and a silastic tube (Silascon No. 00, internal diameter $0.5 \mathrm{~mm}$, external diameter $1.0 \mathrm{~mm}$; Dow Corning Co. Kanagawa, Japan) were implanted into the main mesen- teric lymph duct (Bollman et al. 1948) and the duodenum, respectively, of the acclimatized rats under anaesthesia (pentobarbital sodium, $30 \mathrm{mg} / \mathrm{kg}$ body weight; Abbott, North Chicago, IL, USA). The cannulated rats were kept in Bollman-type restraining cages with a continuous infu- sion $(3 \mathrm{ml} / \mathrm{h})$ of a solution containing L-glucose $(135 \mathrm{mmol} / \mathrm{l})$ and $\mathrm{NaCl}(85 \mathrm{mmol} / \mathrm{l})$. After a $1 \mathrm{~d}$ recovery period, the lymph fluid was collected for $1 \mathrm{~h}$ (the initial value, 0 time); the infusate was then switched to the test lipids ( $100 \mathrm{~g}$ phospholipid/l) emulsified by $10 \mathrm{~g}$ sodium taurocholate/l for $1 \mathrm{~h}$ (administration period) before being 
switched again to the glucose- $\mathrm{NaCl}$ solution. All experiments were done in a room with controlled temperature $\left(22-24^{\circ} \mathrm{C}\right)$, relative humidity $(40-60 \%)$ and lighting (lights on 08.00-20.00 hours). The mesenteric lymph fluid was collected every $2 \mathrm{~h}$ for $8 \mathrm{~h}$ after the switch to the test lipid emulsion. Aortic blood was collected with a heparinized syringe $8 \mathrm{~h}$ after the switch to the test emulsion under a pentobarbital anaesthesia, and the rats were killed. Blood was separated into plasma and blood cells by centrifugation. The blood cells were washed twice with saline $(9 \mathrm{~g} \mathrm{NaCl} / \mathrm{l})$. The small intestine, caecum and the colon were immediately removed with their contents after ligation of both ends of each segment. These were frozen using liquid $\mathrm{N}_{2}$, and stored at $-80^{\circ} \mathrm{C}$ until subsequent analyses.

The present study was approved by the Hokkaido University Animal Committee, and animals were maintained in accordance with the Hokkaido University guidelines for the care and use of laboratory animals.

Test lipids used in experiments were a purified soyabean phosphatidylcholine (SPL, $980 \mathrm{~g}$ soyabean lecithin $/ \mathrm{kg}$, EPIKURON200; Lucus Meyer GmbH, Hamburg, Germany) and brain phospholipids (BPL) containing more than $200 \mathrm{~g}$ plasmalogen $/ \mathrm{kg}$. Brain lipid was extracted from the bovine brain (brain glycerophospholipids) by the method of Folch (1942) with some modifications. The plasmalogen and total phospholipid contents in the extracted BPL fractions were 224 and $821 \mathrm{~g} / \mathrm{kg}$, respectively.

\section{Analytical methods}

Total lipids in the lymphatic fluid, blood plasma and cells, and gastrointestinal contents were extracted using chloroform-methanol-saline (10:5:3, by vol.) (Folch et al. 1957). Plasmalogen was measured by the I addition method (Gottfried \& Rapport, 1962; Williams et al. 1962; Huque et al. 1987), in which the vinyl-ether double bonds in plasmalogen specifically react to $I$ in the presence of methanol. I consumption by the vinyl-ether moiety was measured photometrically at $355 \mathrm{~nm}$. Plasmalogen concentrations evaluated by the I addition method were closely correlated with the values obtained by the p-nitrophenylhydrazone method (Pries \& Böttcher, 1965; $r$ 0.984, data not shown), in which plasmalogen was degraded to fatty aldehyde with sulfuric acid ( $0.3 \mathrm{~mol} / \mathrm{l})$. Fatty aldehydes in the blood plasma and small intestine were measured using the $p$-nitrophenylhydrazone method with sulfuric acid $(5 \mathrm{mmol} / \mathrm{l})$. The specificity of the I addition method for the vinyl-ether moiety in plasmalogen was very high; that is, I consumption by soyabean lecithin and cholesterol was just 0.14 and $0.07 \mathrm{~mol} \%$, respectively, of that by plasmalogen.

Phospholipid content in the extracted lipid fraction was measured by phosphate (Phospholipid-test Wako; Wako Pure Chemical Industries, Osaka, Japan), and triacylglycerol content was assayed by an enzymic procedure (Triglyceride G-test Wako; Wako Pure Chemical Industries).

\section{Calculations and statistical analysis}

We calculated the molar amounts of plasmalogen and phospholipids using $777 \cdot 3,770 \cdot 1$ and 729.5 as the average molecular weights of phospholipids in SPL, BPL and plasmalogen in BPL, respectively.

Plasmalogen absorption rate shown in Table 1 was estimated from the disappearance rate of the lipids from

Table 1. Plasmalogen and phospholipid contents in each part of the alimentary tract and plasmalogen levels of blood $8 \mathrm{~h}$ after the infusion of a lipid emulsion containing soyabean phospholipids (SPL) or brain phospholipids (BPL)

(Mean values and standard errors of the mean)

\begin{tabular}{|c|c|c|c|c|c|}
\hline & \multirow{2}{*}{$\begin{array}{c}\text { Infused } \\
\text { phospholipids }\end{array}$} & \multicolumn{2}{|c|}{ Plasmalogen } & \multicolumn{2}{|c|}{ Phospholipids } \\
\hline & & Mean & SEM & Mean & SEM \\
\hline \multirow[t]{2}{*}{ Small intestine $(\mu \mathrm{mol}) \dagger$} & SPL & $1 \cdot 18$ & 0.22 & $40 \cdot 6$ & $5 \cdot 3$ \\
\hline & BPL & $3 \cdot 65^{\star}$ & 0.77 & $39 \cdot 9$ & $3 \cdot 6$ \\
\hline \multirow[t]{2}{*}{ Caecum ( $\mu \mathrm{mol})$} & SPL & $2 \cdot 30$ & 0.30 & $50 \cdot 7$ & $5 \cdot 6$ \\
\hline & $\mathrm{BPL}$ & $35 \cdot 3^{\star *}$ & 4.5 & $160^{\star \star}$ & $28 \cdot 5$ \\
\hline \multirow[t]{2}{*}{ Colon $(\mu \mathrm{mol})$} & SPL & 0.39 & 0.16 & $4 \cdot 2$ & 1.4 \\
\hline & BPL & $4 \cdot 10$ & 2.57 & $17 \cdot 6$ & $11 \cdot 8$ \\
\hline \multirow[t]{2}{*}{ Total $(\mu \mathrm{mol}) \ddagger$} & SPL & 3.90 & 0.34 & 95.5 & 9.07 \\
\hline & BPL & $42 \cdot 9^{\star *}$ & 1.97 & $217^{\star \star}$ & $18 \cdot 1$ \\
\hline Absorption rate $(\%) \S$ & $\mathrm{BPL}$ & 53.4 & $2 \cdot 10$ & & \\
\hline \multirow[t]{2}{*}{ Blood plasma (nmol/l) } & SPL & $25 \cdot 4$ & $2 \cdot 8$ & & \\
\hline & BPL & $42 \cdot 5 \|$ & $10 \cdot 5$ & & \\
\hline \multirow[t]{2}{*}{ Blood cells (nmol/l) } & SPL & 460 & 103 & & \\
\hline & BPL & 614 & 106 & & \\
\hline
\end{tabular}

Mean values for the SPL and BPL groups were significantly different by the Mann-Whitney test $\left({ }^{*} P<0.05\right.$; $\left.{ }^{\star \star} P<0.01, n 5\right)$.

† Values for the small intestine, but not for the caecum and colon, contain mucosal lipids.

$¥$ Total values are the sum of the amounts of each lipid in the three intestinal segments.

$\S$ Absorption rate of plasmalogen in BPL was estimated as disappearance rate of the vinyl-ether double bonds from the intestines. Details are shown on p. 29.

$\|$ There was a tendency for higher values in the BPL group than in the SPL group by the Mann-Whitney test $(P=0.076)$. 
the alimentary tract using the following equation:

$$
\begin{aligned}
\operatorname{Absorptive} \text { rate }(\%)= & (\text { ingested plasmalogen }(\mu \mathrm{mol}) \\
& - \text { remaining plasmalogen }(\mu \mathrm{mol})) \\
& /(\text { ingested plasmalogen }(\mu \mathrm{mol})) \times 100 .
\end{aligned}
$$

The value of remaining plasmalogen was evaluated by subtracting the mean value of total content (the sum of three intestinal segments) in the SPL group from the total value of each rat in the BPL group.

The results were analysed by two-way ANOVA (Fig. 1). Duncan's multiple-range test was used to determine whether the mean values were significantly different $(P<0.05)$ (Duncan, 1995). The Mann-Whitney test was used in Table 1. These statistical analyses were done by SAS (SAS version 6.07; SAS Institute Inc., Cary, NC, USA).

\section{Results}

Final body weight in groups infused with SPL and BPL were 306 (SEM 5.0) and 306 (SEM 6.0) g, respectively.

Plasmalogen output in the mesenteric lymph was increased $2 \mathrm{~h}$ or later after the switch to BPL emulsion, and reached a peak value at $4-6 \mathrm{~h}$ after the start of BPL infusion (Fig. 1 (a)). Plasmalogen output was not increased after SPL infusion. Total output of plasmalogen for $8 \mathrm{~h}$ (net increase) was 198 (SEM 51.4$) \mathrm{nmol} / 8 \mathrm{~h}$, which is just $0.215 \mathrm{~mol} \%$ of the total infused plasmalogen. The amount of plasmalogen infused was $92 \mu \mathrm{mol}$, and total phospholipids were $320 \mu \mathrm{mol}$ in the BPL group and $378 \mu \mathrm{mol}$ in the SPL group. Lymphatic output of total phospholipids was immediately increased after the infusion of SPL, but not after the infusion of BPL (Fig. 1 (b)). Output reached a peak value at $2-4 \mathrm{~h}$ after the switch in the SPL group, and at $4-6 \mathrm{~h}$ in the BPL group. The net phospholipids increase in the SPL group was more than 3-fold higher than that in the BPL group.
The plasmalogen and phospholipid levels in the caecal contents were much higher in the BPL group than in the SPL groups (Table 1). Plasmalogen absorption rate (disappearance rate) estimated from the lipids remaining in the whole alimentary tract was approximately $50 \mathrm{~mol} \% 8 \mathrm{~h}$ after the administration of BPL.

Plasmalogen concentration in the blood plasma $8 \mathrm{~h}$ after the infusion of test emulsion was $67 \%$ higher in the BPL group than in the SPL group $(P<0.076$; Table 1$)$. There were no differences in long-chain fatty aldehyde levels in both the blood plasma and the small intestine with their luminal contents between the SPL and BPL groups (data not shown).

\section{Discussion}

The present study showed that enterally infused plasmalogen was released into the mesenteric lymph, and change in the lymphatic plasmalogen profile was similar to that of total phospholipid output after an infusion of BPL. These results suggest that lipid-bearing vinyl-ether double bonds are absorbed as a phospholipid. The net increase in plasmalogen output in the lymph was approximately $0.2 \mu \mathrm{mol}$ for $8 \mathrm{~h}$, which is much lower than the total amount of infused plasmalogen $(92 \mu \mathrm{mol}$, recovery rate $0.22 \%)$. In contrast, the disappearance rate of plasmalogen from the whole gut was $54.3 \%$, which is much higher than the lymphatic recovery rate.

Lymphatic recovery rate of the acyl groups in the infused SPL was $74.7 \%$ (infused acyl groups in phosphatidylcholine, $378 \mu \mathrm{mol} \times 2$; net increases in acyl groups in phospholipids, $46.9 \mu \mathrm{mol} \times 2$ (Fig. 1 (b)); in triacylglycerols, $157 \mu \mathrm{mol} \times 3$ (Fig. 1 (c))). The lymphatic triacylglycerol output may be derived from the acyl groups (fatty acids) of soyabean phosphatidylcholine. The reason for the low recovery of the lymph plasmalogen is not the low collection rate of the mesenteric fluid, but the degradation of plasmalogen in the small intestine.
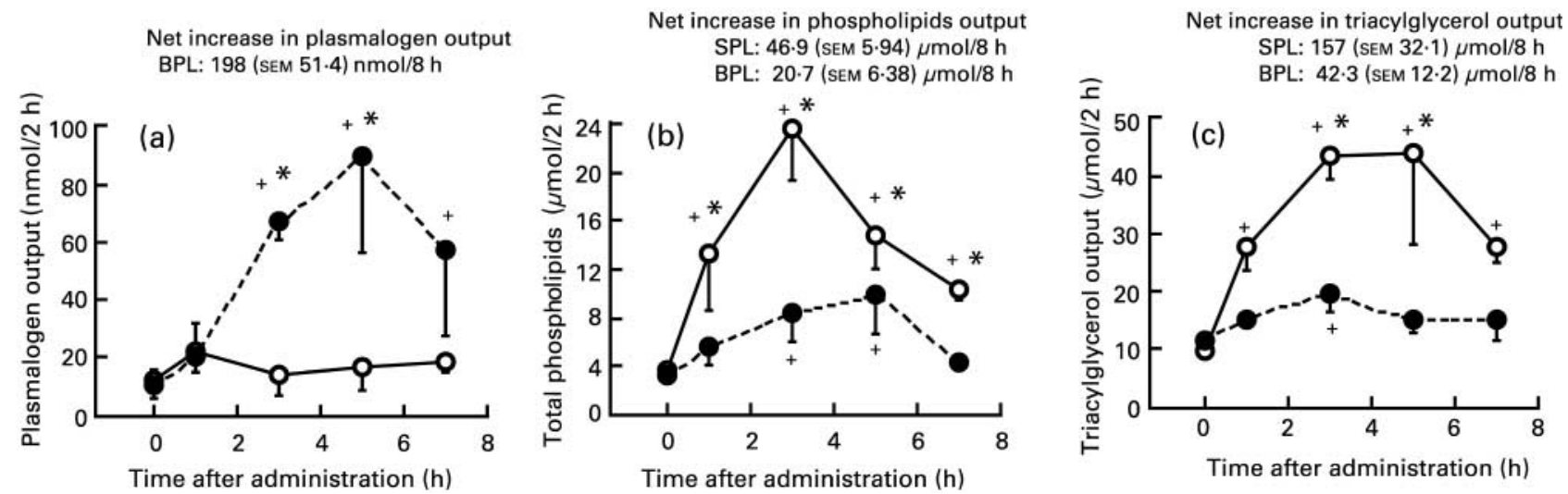

Fig. 1. Lymphatic output of plasmalogen (vinyl-ether double bonds; (a), total phospholipids (b) and triacylglycerols (c) after a duodenal infusion of soyabean phosphatidylcholine (SPL; $\left.378 \mu \mathrm{mol})(-)_{-}\right)$or brain phospholipids (BPL) (-๑-) in mesenteric lymph-cannulated rats. Mean values are shown, with standard errors of the mean represented by vertical bars $(n 5)$. * Mean values were significantly different between the SPL and BPL groups at the same time. Significant increases against the values at time 0 in each group are shown by $+(P<0.05)$. Each point represents collection of the lymph for a $2 \mathrm{~h}$ period; for example, the point at $1 \mathrm{~h}$ shows the values for the $0-2 \mathrm{~h}$ collection. Values at time 0 represent the $2 \mathrm{~h}$ collection before the infusion of the test lipid emulsion. The net increases in output above the graphs show the total lymph output subtracted from the basal (time 0 ) values for $8 \mathrm{~h}$ after the start of a test emulsion. $P$ values for (a) estimated by two-way ANOVA were $P=0.002$ for Lipid (P), $P=0.072$ for Time (T), $P=0.073$ for $P \times T$; for (b) they were $P<0.001$ for Lipid (P), $P<0.001$ for Time $(\mathrm{T}), P=0.002$ for $\mathrm{P} \times \mathrm{T}$; for (c) they were $P<0.001$ for Lipid $(\mathrm{P}), P=0.007$ for Time $(\mathrm{T}), P=0.098$ for $\mathrm{P} \times \mathrm{T}$. 
Plasmalogens are degraded into fatty aldehydes at the $s n-1$ vinyl-ether double bond by lysoplasmalogenase (EC 3.3.2.2) (Jurkowitz-Alexander et al. 1989; Jurkowitz et al. 1999). Oxygen radicals also degrade the vinyl-ether moiety and produce long-chain fatty aldehydes (Stadelmann-Ingrand et al. 2001). However, any increase in the long-chain fatty aldehydes in the lymph, blood plasma and the intestinal tissues was not observed (data not shown), which suggests that the very low recovery of lymphatic plasmalogen was not associated with increases in the cleavage products of vinyl-ether bonds. There is a possibility that saturation of the vinyl-ether double bonds occurs in the intestine. Further study is therefore needed to clarify this activity.

We observed a tendency towards an increase in blood plasmalogen levels. Although the main mesenteric lymph duct was obstructed to collect the lymph fluid, there are a few minor mesenteric lymph ducts. The finding that BPL infusion increased the blood level of plasmalogen by an average of $70 \%$ is important because plasmalogen in blood plasma has been proposed as a lipidaemic antioxidant of lipoproteins (Jurgens et al. 1995; Hahnel et al. 1999). The increase in blood plasmalogen is expected to be much more without obstruction of the main mesenteric lymph duct because about $200 \mathrm{nmol}$ plasmalogen was absorbed, which was much higher than the plasma level before administration. The rate of plasmalogen release into the lymph was very low; however, it may have been sufficient to increase the plasmalogen level in the blood plasma, and for the prevention of CHD or other illness related to oxidative stresses.

We have previously shown that most plasmalogen included in the diet was saved from gastric acid degradation ( $\mathrm{H}$ Hara and $\mathrm{T}$ Wakisata, unpublished results). The present study reveals that dietary plasmalogen is absorbed and released into the lymph as a phospholipid.

\section{References}

American Institute of Nutrition (1977) Report of the American Institute of Nutrition ad hoc Committee on Standards for Nutritional Studies. J Nutr 107, 1340-1348.

American Institute of Nutrition (1980) Second report of the ad hoc Committee on Standards for Nutritional Studies. J Nutr 110, 1726.

Blank ML, Cress EA, Smith ZL \& Snyder F (1992) Meats and fish consumed in the American diet contain substantial amounts of ether-linked phospholipids. J Nutr 122, 1656-1661.

Bollman JL, Cain JC \& Grindlay JH (1948) Techniques for the collection of lymph from the liver, small intestine, or thoracic duct of the rat. $J$ Lab Clin Med 33, 1349-1352.

Duncan DB (1995) Multiple range and multiple $F$ tests. Biometrics 11, 1-42.

Engelmann B, Brautigam C \& Thiery J (1994) Plasmalogen phospholipids as potential protectors against lipid peroxidation of low density lipoproteins. Biochem Biophys Res Commun 204, $1235-1242$.
Farooqui AA, Rapoport SI \& Horrocks LA (1997) Membrane phospholipid alterations in Alzheimer's disease: deficiency of ethanolamine plasmalogens. Neurochem Res 22, 523-527.

Folch J (1942) Brain cephalin, a mixture of phosphatides. Separation from it of phosphatdylserine, phosphatidylethanolamine, and a fraction containing an inositol phosphatides. J Biol Chem 146, 35-44.

Folch J, Lees M \& Sloane-Stanley GH (1957) A simple method for the isolation and purification of total lipides from animal tissues. J Biol Chem 226, 497-509.

Glaser PE \& Gross RW (1994) Plasmenylethanolamine facilitates rapid membrane fusion: a stopped-flow kinetic investigation correlating the propensity of a major plasma membrane constituent to adopt an HII phase with its ability to promote membrane fusion. Biochemistry 33, 5805-5812.

Gottfried EL \& Rapport MM (1962) The biochemistry of plasmalogens. I. Isolation and characterization of phosphatidal choline, a pure native plasmalogen. J Biol Chem 237, 329-333.

Hahnel D, Beyer K \& Engelmann B (1999) Inhibition of peroxyl radical-mediated lipid oxidation by plasmalogen phospholipids and alpha-tocopherol. Free Radic Biol Med 27, 1087-1094.

Huque T, Brand JG, Rabinowitz JL \& Gavarron FF (1987) A comparative study of three methods for the estimation of total plasmalogens in lingual taste epithelium and other tissues. Comp Biochem Physio B 86B, 135-139.

Jurgens G, Fell A, Ledinski G, Chen Q \& Paltauf F (1995) Delay of copper-catalyzed oxidation of low density lipoprotein by in vitro enrichment with choline or ethanolamine plasmalogens. Chem Phys Lipids 77, 25-31.

Jurkowitz MS, Horrocks LA \& Litsky ML (1999) Identification and characterization of alkenyl hydrolase (lysoplasmalogenase) in microsomes and identification of a plasmalogen-active phospholipase A2 in cytosol of small intestinal epithelium. Biochim Biophys Acta 1437, 142-156.

Jurkowitz-Alexander M, Ebata H, Mills JS, Murphy EJ \& Horrocks LA (1989) Solubilization, purification and characterization of lysoplasmalogen alkenylhydrolase (lysoplasmalogenase) from rat liver microsomes. Biochim Biophys Acta 1002, 203-212.

Nagan N \& Zoeller RA (2001) Plasmalogens: biosynthesis and functions. Prog Lipid Res 40, 199-229.

Pries C \& Böttcher CJF (1965) The determination of free and plasmalogen-bound aldehydes in lipid fraction. Biochim Biophys Acta 98, 329-334.

Stadelmann-Ingrand S, Favreliere S, Fauconneau B, Mauco G \& Tallineau C (2001) Plasmalogen degradation by oxidative stress: Production and disappearance of specific fatty aldehydes and fatty alpha-hydroxyaldehydes. Free Radic Biol Med 31, $1263-1271$.

Williams JNJ, Anderson CE \& Jasik AD (1962) A sensitive and specific method for plasmalogens and other enol esters. J Lipid Res 3, 378-381.

Zoeller RA, Lake AC, Nagan N, Gaposchkin DP, Legner MA \& Lieberthal W (1999) Plasmalogens as endogenous antioxidants: somatic cell mutants reveal the importance of the vinyl-ether. Biochem J 338, 769-776.

Zoeller RA, Morand OH \& Raetz CR (1988) A possible role for plasmalogens in protecting animal cells against photosensitized killing. J Biol Chem 263, 11590-11596.

Zommara M, Tachibana N, Mitsui K, et al. (1995) Inhibitory effect of ethanolamine plasmalogen on iron- and copper-dependent lipid peroxidation. Free Radic Biol Med 18, 599-602. 